\title{
State protection by quantum control before and after noise processes
}

\author{
Hiroaki Wakamura, ${ }^{1, *}$ Ryûitirô Kawakubo, ${ }^{1, \dagger}$ and Tatsuhiko Koike ${ }^{1,2, \ddagger}$ \\ ${ }^{1}$ Department of Physics, Keio University, Yokohama 223-8522, Japan \\ ${ }^{2}$ Research and Education Center for Natural Sciences, Keio University, Yokohama 223-8521, Japan
}

(Received 9 April 2017; published 29 August 2017)

\begin{abstract}
We discuss protection of a quantum state that goes through a noise process by measurements and operations before and after the noise process. In our previous work, we showed the nonexistence of "truly quantum" protocols that protect an unknown qubit state against depolarizing noise better than "classical" ones. Toward identifying the class of noise processes that is optimally suppressed by such a "classical" protocol, we extend our previous result in two directions. First, we show that the statement is also true in any finite-dimensional Hilbert spaces, which was previously conjectured; the optimal protocol is either the do nothing protocol or the discriminate and reprepare protocol, depending on the strength of the noise. Second, in the case of a single qubit, we show that essentially the same conclusion holds for any unital noise. Thus, the noise must be nonunital for a control protocol beyond "classical" ones to exist.
\end{abstract}

DOI: 10.1103/PhysRevA.96.022325

\section{INTRODUCTION}

Quantum information technology, such as quantum computation, quantum cryptography, etc., is a new framework of information processing where quantum states (e.g., qubits) bear information in place of classical bits. One of the difficulties in the realization of those technologies is the existence of noise. Since there is no isolated physical system in the world, the state inevitably undergoes noise processes caused by interactions with the environment. The state evolution becomes irreversible and errors occur in information processing. In order to reduce errors and make information processing feasible, the protection of quantum states is an important task [1-5].

In the classical world, one can in principle protect any state against any noise, by taking the complete record of the state before the noise affects the system. In the quantum world, it is not the case even if the state is not a probabilistic mixture. If one could do so, then one could suppress the disturbance caused by measurements and realize disturbance-free measurements. This would contradict quantum measurement theory [6,7], which implies that quantum measurements cannot extract the full information from a single sample and inevitably disturb the state. Thus impossibility of perfect state protection reflects the nature of quantum mechanics, in the same way as the impossibility of perfect state discrimination [8-11] or quantum cloning [12].

Given this impossibility of perfect state protection, one may still want to consider control protocols which suppress the noise approximately. This is similar to pursuing the error-disturbance uncertainty relation $[13,14]$ or the theory of imperfect cloning $[15,16]$. Quantitative analysis of the limits

\footnotetext{
*hwakamura@rk.phys.keio.ac.jp

${ }^{\dagger}$ rkawakub@rk.phys.keio.ac.jp

${ }^{\ddagger}$ koike@ phys.keio.ac.jp
}

Published by the American Physical Society under the terms of the Creative Commons Attribution 4.0 International license. Further distribution of this work must maintain attribution to the author(s) and the published article's title, journal citation, and DOI. in state protection may reveal the role played by measurements in state protection and whether there exists a comprehensive point of view to achieve optimal state protection. That may also clarify the fundamental limitations in our ability to manipulate quantum systems and provide an operational characterization of the quantum world.

We would like to refer to recent works in the context of the ex-ante-ex-post control scheme [17]. The scheme consists of a general measurement before the noise process (ex-ante control), and operations after the noise process depending on the outcomes of the measurements (ex-post control), as depicted in Fig. 1. Branczyk et al. [18] and Mendonça et al. [19] have derived an optimal protocol which protects two states of a qubit solely by ex-post control. Their protocol detects the influence of noise without discriminating the input states at all. This is an example where it is beneficial to extract information after the noise process. On the other hand, when the input is a completely unknown pure state, Zhang et al. [20] showed that one cannot suppress depolarizing noise at all by ex-post control alone. The present authors, in the qubit case, extended their results to general noise [17]: A unitary operation is the optimal ex-post control protocol to protect a completely unknown pure state against arbitrary noise, i.e., it is never beneficial to extract information in ex-post control.

All these results suggest that prior knowledge of the input state in some form is essential for its protection. Thus, if the input state is completely unknown, one is led to consider ex-ante control. Ex-ante control was considered previously by Korotokov and Keane [21] and then by Wang et al. [22]. Although their interests are protocols with postselection, one can find in the numerical results of Ref. [22] that there is a nontrivial ex-ante-ex-post control protocol (without postselection) which suppresses amplitude damping noise. In contrast, the present authors [17] proved that nontrivial ex-ante-ex-post control cannot suppress depolarizing noise better than "classical" protocols. More precisely, the optimal protocols are the "do nothing" and the "discriminate and reprepare" protocols, where the latter consists of an ex-ante projection measurement and an ex-post repreparation of the state corresponding to the outcome. Thus, while amplitude damping noise can be suppressed by a nontrivial quantum 


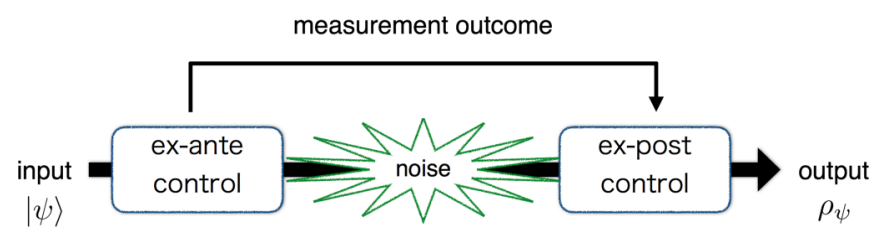

FIG. 1. Schematic diagram of quantum control (ex-ante control and ex-post control).

protocol, depolarizing noise cannot. Then it is natural to ask which class of noise allows or disallows nontrivial quantum state protection.

In this paper, we extend our previous results in Ref. [17] in two directions. The first direction is to extend the results on depolarizing noise to higher-dimensional Hilbert spaces. We prove that noise is optimally suppressed either by the do nothing protocol or by the discriminate and reprepare protocol, which was conjectured in Ref. [17]. The second direction is to widen the types of noise in the case of a qubit. We will study the class of unital noise, which leaves the completely mixed state unchanged and is considered to be "unbiased." It can be said that unital noise makes any state more random because it never decrease the (von Neumann) entropy of a quantum state. This class contains many types of noise appearing in quantum information, including depolarizing noise, dephasing noise, and bit-flip noise, but does not contain amplitude damping noise. We show that the optimal ex-ante-ex-post control protocol to suppress unital noise is either a no measurement protocol or the discriminate and reprepare protocol. It is proved that unital noise is contained in the class of noise which is optimally suppressed by classical protocols.

To show the above results, we introduce a method which differs from that of Ref. [17]. This method is based on general observations which help us to understand the structure of the problem more clearly. One is on the property of so-called quantum-classical-quantum channels. The other is on the structure (convexity) of the space of noise that is optimally suppressed by a single protocol. In our discussion, the noise that is optimally suppressed by two or more protocols will play a significant role.

The paper is organized as follows. After a brief review of the basic mathematical tools in Sec. II, the state protection scheme by ex-ante-ex-post control is introduced in Sec. III. We give general observations on noise suppression in Sec. IV. In Sec. IV, we show our first main result, that "classical" protocols suffice in state protection against depolarizing noise. Then, we focus on the case of a single qubit. We review the geometry of the space of unital noise in Sec. VI. In Sec. VII, we show our second main result, that "classical" protocols are optimal for unital noise. Section VIII is devoted to conclusion and discussions.

\section{BASICS OF QUANTUM OPERATIONS}

We shall introduce the basic mathematical tools and notation used in our analysis. Throughout the paper, we consider physical systems which are represented by a finite-dimensional Hilbert space. Let $\mathcal{H}$ be such a Hilbert space and $\mathcal{L}(\mathcal{H})$ be the set of all linear operators on $\mathcal{H}$. A quantum state is described by a density operator $\rho \in \mathcal{L}(\mathcal{H})$ such that $\rho \geqslant 0$ and $\operatorname{Tr} \rho=1$. Since the control of quantum states consists of measurement and operations, the mathematical map corresponding to measurement or operations is explained below.

Any physical evolution of a quantum state corresponds to a trace-preserving completely positive (TPCP) map, and vice versa (e.g., Ref. [23]). Here, a linear map $\mathcal{E}: \mathcal{L}(\mathcal{H}) \rightarrow$ $\mathcal{L}(\mathcal{H})$ is said to be positive if $X \geqslant 0$ implies $\mathcal{E}(X) \geqslant 0$ and completely positive $(C P)$ if the map $\mathcal{E} \otimes \mathrm{id}_{n}$ is positive for every positive integer $n$, where $\mathrm{id}_{n}$ denotes the identity map on $\mathcal{L}\left(\mathbb{C}^{n}\right)=\mathbb{C}^{n \times n}$. The map $\mathcal{E}$ is said to be trace preserving $(T P)$ if $\operatorname{Tr} \mathcal{E}(X)=\operatorname{Tr} X$ for any $X \in \mathcal{L}(\mathcal{H})$.

Any physical measuring process corresponds to a $\mathrm{CP}$ instrument, and vice versa [7]. Here, a $C P$ instrument is a family $\left\{\mathcal{J}_{\omega}\right\}_{\omega \in \Omega}$ of CP maps with $\sum_{\omega \in \Omega} \mathcal{J}_{\omega}$ being trace preserving. We assume that the set $\Omega$ of outcomes is finite throughout the paper. The state evolution by the measurement is described as

$$
\rho \mapsto \frac{\mathcal{J}_{\omega}(\rho)}{\operatorname{Tr} \mathcal{J}_{\omega}(\rho)}, \quad \text { with probability } \quad \operatorname{Tr} \mathcal{J}_{\omega}(\rho) .
$$

The probability distribution of measurement outcomes is described by a positive-operator-valued measure (POVM), which is a family $\left\{M_{\omega}\right\}_{\omega \in \Omega}$ of positive operators on $\mathcal{H}$ such that $\sum_{\omega \in \Omega} M_{\omega}$ is the identity operator. A CP instrument $\left\{\mathcal{J}_{\omega}\right\}_{\omega \in \Omega}$ defines a POVM $\left\{M_{\omega}\right\}_{\omega \in \Omega}$ by $\operatorname{Tr} \rho M_{\omega}=\operatorname{Tr} \mathcal{J}_{\omega}(\rho)$ or $M_{\omega}=\mathcal{J}_{\omega}^{*}(1)$, where an asterisk denotes the dual map. The dual map $\mathcal{E}^{*}$ of $\mathcal{E} \in \mathcal{L}(\mathcal{L}(\mathcal{H}))$ is defined by $\operatorname{Tr} \mathcal{E}(X) Y=\operatorname{Tr} X \mathcal{E}^{*}(Y)$. We shall say that a POVM $\left\{M_{\omega}\right\}_{\omega \in \Omega}$ and a CP instrument $\left\{\mathcal{J}_{\omega}\right\}_{\omega \in \Omega}$ above are associated with each other. A POVM has all the information on the statistical properties of the measurement outcomes, while a CP instrument $\left\{\mathcal{J}_{\omega}\right\}$ has further information on the resulting states after the measurement.

The space $\mathcal{L}(\mathcal{H})$ of linear operators can be regarded as a Hilbert space with the Hilbert-Schmidt inner product $\langle X, Y\rangle_{\mathrm{HS}}:=\operatorname{Tr} X^{\dagger} Y$. A linear map $\mathcal{E}$ on $\mathcal{L}(\mathcal{H})$ is interpreted as a linear operator on the Hilbert space $\mathcal{L}(\mathcal{H})$. The trace of such $\mathcal{E}$ is defined by

$$
\operatorname{Tr}_{\mathrm{HS}} \mathcal{E}:=\sum_{i}\left\langle V_{i}, \mathcal{E}\left(V_{i}\right)\right\rangle_{\mathrm{HS}}
$$

where $\left\{V_{i}\right\}_{i}$ is an orthonormal basis of the Hilbert space $\mathcal{L}(\mathcal{H})$. For example, when $\operatorname{dim} \mathcal{H}=2$, an orthonormal basis of $\mathcal{L}(\mathcal{H})$ is given by $\left\{\sigma_{\mu} / \sqrt{2}\right\}_{\mu=0}^{3}$, where $\sigma_{0}$ is the identity operator, and $\sigma_{i}, 1 \leqslant i \leqslant 3$, are the Pauli operators. Then the trace is written as

$$
\operatorname{Tr}_{\mathrm{HS}} \mathcal{E}=\frac{1}{2} \sum_{\mu=0}^{3} \operatorname{Tr}\left[\sigma_{\mu} \mathcal{E}\left(\sigma_{\mu}\right)\right]
$$

\section{THE SETUP}

We shall discuss the state protection in the ex-ante-expost quantum control scheme below, which was proposed in Ref. [17] (see Fig. 1). The scheme consists of the following:

(1) State preparation: An unknown state is prepared.

(2) Ex-ante control: A measurement is performed, which is described by a CP instrument $\left\{\mathcal{J}_{\omega}\right\}_{\omega \in \Omega}$.

(3) Noise: The state undergoes an undesired evolution, called "noise," described by a TPCP map $\mathcal{N}$. 
(4) Ex-post control: An operation, which depends on the measurement outcome $\omega$ of the ex-ante control, is performed on the system. This is described by a family $\left\{\complement_{\omega}\right\}_{\omega \in \Omega}$ of TPCP maps.

For given noise $\mathcal{N}$, an ex-ante-ex-post control protocol is specified by the family $\left\{\left(\mathcal{J}_{\omega}, \mathrm{C}_{\omega}\right)\right\}_{\omega \in \Omega}$. As we did in Ref. [17], we focus on the case where the states prepared in step (1) are pure and are completely unknown, i.e., the prior probability distribution is uniform on the unit sphere in $\mathcal{H}$, though one can consider more general cases within the scheme above. Our problem is to find an optimal ex-ante-ex-post control protocol $\left\{\left(\mathcal{J}_{\omega}, \mathcal{C}_{\omega}\right)\right\}_{\omega \in \Omega}$ for given noise $\mathcal{N}$ such that the states after the measurement with outcome $\omega$ are as close to the original state $|\psi\rangle\langle\psi|$ as possible. We evaluate the closeness by fidelity, which is expressed by $F(\rho,|\psi\rangle\langle\psi|):=\langle\psi|\rho| \psi\rangle$ if one of the two states is pure (e.g., Ref. [23]), and the optimality is defined by the average fidelity with respect to the probability to obtain each outcome $\omega$ and with respect to that of each input state $|\psi\rangle$.

An advantage of the choice is that the resulting averaged evaluation function, the average fidelity

$$
\bar{F}=\int_{\||\psi\rangle \|=1} d \psi\langle\psi|\mathcal{E}(|\psi\rangle\langle\psi|)| \psi\rangle,
$$

depends on the average operation

$$
\mathcal{E}:=\sum_{\omega=1}^{M} \mathcal{C}_{\omega} \circ \mathcal{N} \circ \mathcal{J}_{\omega},
$$

which is a TPCP map. We will use the formula [17]

$$
\bar{F}=\frac{d+\operatorname{Tr}_{\mathrm{HS}} \mathcal{E}}{d(d+1)},
$$

where $d:=\operatorname{dim} \mathcal{H}$ and $\mathcal{E}$ is the average operation (5) of the protocol $\left\{\left(\mathcal{J}_{\omega}, \mathrm{C}_{\omega}\right)\right\}$.

\section{TWO GENERAL OBSERVATIONS}

In this section, we give two general observations on noise suppression problem. They will be the basis for the main results of the paper, Theorems 1 and 2 .

First, we give a sufficient condition for the noise to be optimally suppressed by the discriminate and reprepare protocol (Proposition 1). Let $\mathcal{H}$ be a $d$-dimensional Hilbert space. Let us consider operations of the form

$$
\mathcal{F}(\rho)=\sum_{k} \rho_{k} \operatorname{Tr} M_{k} \rho .
$$

Physically, one measures the input state $\rho$ by a POVM $\left\{M_{k}\right\}$ and prepares a state $\rho_{k}$ according to the measurement outcome $k$. Such an operation is called an quantum-classical-quantum (QCQ) channel [24-26].

In order to suppress a given QCQ noise, we consider the discriminate and reprepare protocol $\left\{\left(\mathcal{J}_{\omega}, \mathrm{C}_{\omega}\right)\right\}_{1 \leqslant \omega \leqslant d}(\omega$ is an integer) that is defined by

$$
\begin{gathered}
\mathcal{J}_{\omega}(\rho)=\left|\phi_{\omega}\right\rangle\left\langle\phi_{\omega}|\rho| \phi_{\omega}\right\rangle\left\langle\phi_{\omega}\right|, \\
\mathcal{C}_{\omega}(\rho)=\left|\phi_{\omega}\right\rangle\left\langle\phi_{\omega}\right| \operatorname{Tr} \rho,
\end{gathered}
$$

where $\left\{\left|\phi_{\omega}\right\rangle\right\}_{1 \leqslant \omega \leqslant d}$ is an arbitrary orthonormal basis of $\mathcal{H}$. Physically, one discriminates the input state between certain $d$ orthogonal states $\left\{\left|\phi_{\omega}\right\rangle\right\}_{1 \leqslant \omega \leqslant d}$ and reprepares the discriminated state $\left|\phi_{\omega}\right\rangle$ after the noise process. The average fidelity is easily calculated as

$$
\bar{F}_{\mathrm{DR}}=\frac{2}{d+1} .
$$

Indeed, it follows from (9) that $\mathcal{C}_{\omega} \circ \mathcal{N} \circ \mathcal{J}_{\omega}=\mathcal{C}_{\omega} \circ \mathcal{J}_{\omega}$ holds for any trace-preserving $\mathcal{N}$. From Eq. (3), one has

$$
\operatorname{Tr}_{\mathrm{HS}} \mathrm{C}_{\omega} \circ \mathcal{J}_{\omega}=\frac{1}{2} \operatorname{Tr} \mathcal{J}_{\omega} \circ \mathrm{C}_{\omega}(1)=1 .
$$

Then, the average fidelity (10) is obtained via formula (6) for $\bar{F}$. We note that the fidelity is independent from $\mathcal{N}$ and from the choice of $\left\{\left|\phi_{\omega}\right\rangle\right\}$.

Proposition 1. Any QCQ noise is optimally suppressed by the discriminate and reprepare protocol $\left\{\left(\mathcal{J}_{\omega}, \mathrm{C}_{\omega}\right)\right\}_{1 \leqslant \omega \leqslant d}$ defined by (8) and (9). The optimal average fidelity is given by (10).

Proof. First, we observe that if $\mathcal{N}$ is a QCQ channel, then the average operation $\mathcal{E}=\sum_{\omega} \mathcal{C}_{\omega} \circ \mathcal{N} \circ \mathcal{J}_{\omega}$ for any protocol $\left\{\left(\mathcal{J}_{\omega}, \mathcal{C}_{\omega}\right)\right\}$ is also a QCQ channel. Indeed, if $\mathcal{N}$ is written in the form (7), then one has

$$
\mathcal{E}(\rho)=\sum_{\omega, k} \mathrm{C}_{\omega}\left(\rho_{k}\right) \operatorname{Tr}\left[\mathcal{J}_{\omega}^{*}\left(M_{k}\right) \rho\right],
$$

where $\mathcal{C}_{\omega}\left(\rho_{k}\right)$ is a state and $\left\{\mathcal{J}_{\omega}^{*}\left(M_{k}\right)\right\}$ is a POVM. Second, we recall that the maximum average fidelity between the input and output states of a QCQ channel is given by $\bar{F}=2 /(d+1)$ [27]. Therefore, the average fidelity $\bar{F}$ for any protocol $\left\{\left(\mathcal{J}_{\omega}, \mathrm{C}_{\omega}\right)\right\}$ does not exceed that value. On the other hand, the value can be attained by the discriminate and reprepare protocol (8) and (9).

The proposition above solves the problem of state protection against a QCQ noise. Several equivalent conditions for a map to be QCQ are known [26]. For example, a QCQ channel is entanglement breaking, i.e., it breaks the entanglement between the transmitted system and any other system. We make use of one of the equivalent conditions in the proof of Theorem 1.

Next, we give our second observation, which is about convexity. It is quite general and provides us a clear understanding of the structure of the problem.

Proposition 2. The space of all noise channels that are optimally suppressed by a single ex-ante-ex-post control protocol $\left\{\left(\mathcal{J}_{\omega}, \mathrm{C}_{\omega}\right)\right\}_{\omega \in \Omega}$ is convex.

Proof. The claim is equivalent to the following: If a control protocol $\left\{\left(\mathcal{J}_{\omega}, \mathrm{C}_{\omega}\right)\right\}$ optimally suppresses two noise channels $\mathcal{N}_{1}$ and $\mathcal{N}_{2}$, then it also optimally suppresses any mixture of them, $\mathcal{N}:=(1-\alpha) \mathcal{N}_{1}+\alpha \mathcal{N}_{2}, 0 \leqslant \alpha \leqslant 1$. Let $\left\{\left(\mathcal{J}_{\omega}, \mathrm{C}_{\omega}\right)\right\}$ be such a protocol. By (5) and (6), the optimal protocol maximizes $\operatorname{Tr}_{\text {HS }} \mathcal{E}$. For any protocol $\left\{\left(\mathcal{J}_{\omega}^{\prime}, \mathrm{C}_{\omega}^{\prime}\right)\right\}$, one has

$$
\begin{aligned}
& \operatorname{Tr}_{\mathrm{HS}} \mathcal{J}_{\omega}^{\prime} \circ \mathcal{N} \circ \mathrm{C}_{\omega}^{\prime} \\
& \quad=(1-\alpha) \operatorname{Tr}_{\mathrm{HS}} \mathcal{J}_{\omega}^{\prime} \circ \mathcal{N}_{1} \circ \mathrm{C}_{\omega}^{\prime}+\alpha \operatorname{Tr}_{\mathrm{HS}} \mathcal{J}_{\omega}^{\prime} \circ \mathcal{N}_{2} \circ \mathrm{C}_{\omega}^{\prime} \\
& \quad \leqslant(1-\alpha) \operatorname{Tr}_{\mathrm{HS}} \mathcal{J}_{\omega} \circ \mathcal{N}_{1} \circ \mathrm{C}_{\omega}+\alpha \operatorname{Tr}_{\mathrm{HS}} \mathcal{J}_{\omega} \circ \mathcal{N}_{2} \circ \mathrm{C}_{\omega} \\
& \quad=\operatorname{Tr}_{\mathrm{HS}} \mathcal{J}_{\omega} \circ \mathcal{N} \circ \mathrm{C}_{\omega} .
\end{aligned}
$$

Thus $\left\{\left(\mathcal{J}_{\omega}, \mathrm{C}_{\omega}\right)\right\}$ maximizes $\operatorname{Tr}_{\mathrm{HS}} \mathcal{J}_{\omega}^{\prime} \circ \mathcal{N} \circ \mathrm{C}_{\omega}^{\prime}$ so that it optimally suppresses $\mathcal{N}$. 


\section{SUPPRESSION OF DEPOLARIZING NOISE}

We give the first main result, which solves the optimality problem for the depolarizing noise for any $d$-dimensional Hilbert space.

Theorem 1. The optimal ex-ante-ex-post protocol $\left\{\left(\mathcal{J}_{\omega}, \mathrm{C}_{\omega}\right)\right\}_{1 \leqslant \omega \leqslant d}$ for depolarizing noise

$$
\mathcal{N}(\rho)=(1-\varepsilon) \rho+\varepsilon \frac{1}{d} \operatorname{Tr} \rho, \quad 0 \leqslant \varepsilon \leqslant 1,
$$

is given as follows.

(i) When the noise is weak, $\varepsilon \leqslant d /(d+1)$, the do nothing protocol $\left\{\left(\mathcal{J}_{\omega}, \mathrm{C}_{\omega}\right)\right\}_{\omega=1}$ with

$$
\left(\mathcal{J}_{1}, \mathrm{C}_{1}\right)=(\mathrm{id}, \mathrm{id})
$$

is optimal. The optimal average fidelity is $\bar{F}_{\mathrm{DN}}=1-\varepsilon(d-$ 1) $/$ d.

(ii) When the noise is strong, $\varepsilon \geqslant d /(d+1)$, the discriminate and reprepare protocol $\left\{\left(\mathcal{J}_{\omega}, \mathrm{C}_{\omega}\right)\right\}_{1 \leqslant \omega \leqslant d}$ given by (8) and (9) is optimal. The optimal average fidelity is $\bar{F}_{\mathrm{DR}}=$ $2 /(d+1)$.

Proof. We prove (ii) first and (i) later.

(ii) By Proposition 1, it suffices to show that the noise $\mathcal{N}$ is QCQ when $\varepsilon \geqslant d /(d+1)$. In general, a linear map $\mathcal{N}$ is QCQ if and only if the image of the maximally entangled state $|\Psi\rangle:=(1 / \sqrt{d}) \sum_{i}|i i\rangle$ by $\mathrm{id} \otimes \mathcal{N}$ is separable [26]. In the present case, it follows from (14) that

$$
(\mathrm{id} \otimes \mathcal{N})(|\Psi\rangle\langle\Psi|)=(1-\varepsilon)|\Psi\rangle\langle\Psi|+\varepsilon \frac{1}{d} .
$$

The right-hand side of (16) is a mixture of the maximally entangled state and the completely mixed state. Such a state is separable if and only if $\varepsilon \geqslant d /(d+1)$ [see Eq. (41) in Ref. [28]]. Thus, $\mathcal{E}$ is QCQ if and only if $\varepsilon \geqslant d /(d+1)$. This proves (ii).

(i) The do nothing protocol gives the average fidelity

$$
\bar{F}_{\mathrm{DN}}=1-\varepsilon+\frac{\varepsilon}{d},
$$

which can be calculated directly by (4). Consider first the case $\varepsilon=0$. Then the protocol is optimal because it achieves $\bar{F}_{\text {DN }}=1$. Consider next the case $\varepsilon=d /(d+1)$. From (ii), $\mathcal{N}$ is optimally suppressed by the discriminate and reprepare protocol. The do nothing protocol achieves the same average fidelity because one has $\bar{F}_{\mathrm{DN}}=2 /(d+1)$ from (17). The noise channel $\mathcal{N}$ is optimally suppressed also by the do nothing protocol. Thus, both of the noise channels $\mathcal{N}$ with $\varepsilon=0$ and with $\varepsilon=d /(d+1)$ are suppressed by the same do nothing protocol. By Proposition 2, any noise channel $\mathcal{N}$ with $0 \leqslant \varepsilon \leqslant$ $d /(d+1)$, which is a convex combination of those with $\varepsilon=0$ and $\varepsilon=d /(d+1)$, is optimally suppressed by the do nothing protocol.

\section{GEOMETRY OF UNITAL NOISE}

The class of unital noise appears commonly in quantum information. Examples are depolarizing noise, dephasing noise, and bit-flip noise. In particular, dephasing noise, which describes decoherence between energy eigenstates or T2 relaxation, is important in practice. In this section, we describe the geometry of unital noise (e.g., Ref. [29]), which is used in Theorem 2 in the next section.

A linear map $\mathcal{E}$ on operators is said to be unital if it preserves the identity operator, $\mathcal{E}(1)=1$. We can interpret unital TPCP maps as "unbiased," because they keep the completely mixed state. An important characteristic of a unital TPCP map $\mathcal{E}$ is that it never decreases the von Neumann entropy $S(\rho)$ of a quantum state $\rho$, i.e., $S(\mathcal{E}(\rho)) \geqslant S(\rho)$. Thus one can say that unital noise $\mathcal{E}$ always increases (or at least keeps) the randomness of the input state $\rho$. We remark that one way to understand the inequality above is to apply the well-known nonincreasing property of quantum relative entropy $S(\rho \| \sigma):=\operatorname{Tr}[\rho \ln \rho-\rho \ln \sigma]$ under a TPCP map $\mathcal{E}$, i.e., $S(\mathcal{E}(\rho) \| \mathcal{E}(\sigma)) \leqslant S(\rho \| \sigma)$, to the state $\sigma=1 / d$. In this section, we briefly summarize the facts about the convex structure of the space of unital TPCP maps on a qubit.

We consider the set of unital TPCP maps on $\mathcal{L}(\mathcal{H})$. From the definition of unital TPCP maps, it is easy to see that the set is convex in $\mathcal{L}(\mathcal{L}(\mathcal{H}))$, is closed under composition, and contains all unitary operations. If $\operatorname{dim} \mathcal{H}=2$, because each Pauli operator $\sigma_{\mu}$ is unitary, the map

$$
\mathcal{N}=\sum_{\mu=0}^{3} \alpha^{\mu} \mathcal{A}_{\sigma_{\mu}}, \quad \alpha^{\mu} \geqslant 0, \quad \sum_{\mu} \alpha^{\mu}=1,
$$

is unital and TPCP, where $\mathcal{A}_{U}(\rho):=U \rho U^{\dagger}$. It follows that the map

$$
\mathcal{N}^{\prime}=\mathcal{A}_{V} \circ \mathcal{N} \circ \mathcal{A}_{U}
$$

is also unital and TPCP if $U$ and $V$ are unitary operators. Conversely, it is known [29] that the above $\mathcal{N}^{\prime}$ runs over all unital TPCP maps when we vary $\alpha^{\mu}, U$, and $V$. Thus, apart from the degree of freedom of fixed unitary operations on the input and output states, the unital TPCP maps are parametrized by $\alpha^{\mu}$ as in (18). They form a tetrahedron $T$ with vertices at $\alpha^{\mu}=(1,0,0,0),(0,1,0,0),(0,0,1,0)$, and $(0,0,0,1)$. These vertices correspond to unitary operations $\mathcal{A}_{\sigma_{\mu}}$.

For later calculation, we introduce a new coordinate system $\left(d^{i}\right)$ by

$$
\begin{gathered}
{\left[\begin{array}{l}
d^{1} \\
d^{2} \\
d^{3}
\end{array}\right]=\alpha^{0}\left[\begin{array}{l}
1 \\
1 \\
1
\end{array}\right]+\alpha^{1}\left[\begin{array}{c}
1 \\
-1 \\
-1
\end{array}\right]+\alpha^{2}\left[\begin{array}{c}
-1 \\
1 \\
-1
\end{array}\right]} \\
+\alpha^{3}\left[\begin{array}{c}
-1 \\
-1 \\
1
\end{array}\right]
\end{gathered}
$$

so that

$$
\mathcal{N}\left(\sigma_{i}\right)=d^{i} \sigma_{i} \quad(\text { no sum })
$$

holds. In the coordinate system $\left(d^{i}\right)$, the tetrahedron $T$ has the vertices at $\mathcal{E}_{0}(1,1,1), \mathcal{E}_{1}(1,-1,-1), \mathcal{E}_{2}(-1,1,-1)$, and $\varepsilon_{3}(-1,-1,1)$ (Fig. 2). Let $\varepsilon_{\mu \nu}(\mu \neq \nu)$ be the midpoint of $\varepsilon_{\mu}$ and $\varepsilon_{v}$, and let $O$ be the octahedron whose vertices are the six midpoints $\mathcal{E}_{\mu \nu}$. Then, the space $\overline{T \backslash O}$ consists of four smaller tetrahedra. Let $T_{\mu}(\mu=0,1,2,3)$ be each of such tetrahedra that contains $\mathcal{E}_{\mu}$. Thus $T=O \cup T_{0} \cup T_{1} \cup T_{2} \cup T_{3}$. In the following, we identify each unital noise channel represented by (21) [or (18)] with a point in the tetrahedron $T$. 

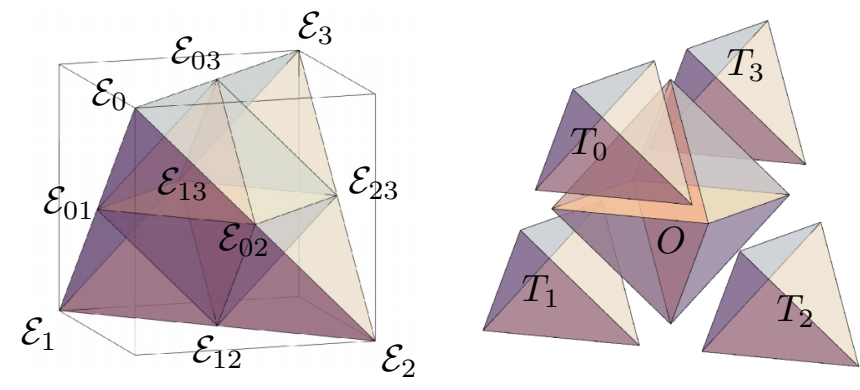

FIG. 2. Left: The space of unital noise channels is (up to certain equivalence) a tetrahedron $T$ with vertices at $\mathcal{E}_{0}(1,1,1)$, $\mathcal{E}_{1}(1,-1,-1), \mathcal{E}_{2}(-1,1,-1)$, and $\mathcal{E}_{3}(-1,-1,1)$, which are unitary operations. A unital noise channel is a convex combination of $\varepsilon_{\mu}$. Right: The tetrahedron $T$ is decomposed into an octahedron $O$ and four tetrahedra $T_{\mu}$. The six vertices of $O$ are the midpoints $\varepsilon_{\mu \nu}$ of the edges, which are $( \pm 1,0,0),(0, \pm 1,0)$, and $(0,0, \pm 1)$. This decomposition will be used in Theorem 2 .

We remark on the tetrahedral symmetry of $T$, which is the remaining symmetry on $T$ caused by the freedom of $U$ and $V$ in (19). A pair $(U, V)$ of unitary operators determines by (19) an automorphism $\mathcal{N} \mapsto \mathcal{N}^{\prime}$ of the convex space of unital TPCP maps. If the pair $(U, V)$ is properly chosen, the automorphism sends the tetrahedron $T$ to itself so that it is a tetrahedral symmetry map. For example, when $(U, V)=$ $\left(1, \sigma_{3}\right)$, the automorphism is $\left(d^{1}, d^{2}, d^{3}\right) \mapsto\left(-d^{1},-d^{2}, d^{3}\right)$ and sends $\left(\mathcal{E}_{0}, \mathcal{E}_{1}, \mathcal{E}_{2}, \mathcal{E}_{3}\right)$ to $\left(\mathcal{E}_{3}, \mathcal{E}_{2}, \mathcal{E}_{1}, \mathcal{E}_{0}\right)$. When $(U, V)=$ $\left(e^{i \pi \sigma_{3} / 4}, e^{-i \pi \sigma_{3} / 4}\right)$, the automorphism is $\left(d^{1}, d^{2}, d^{3}\right) \mapsto$ $\left(d^{2}, d^{1}, d^{3}\right)$ and sends $\left(\mathcal{E}_{0}, \mathcal{E}_{1}, \mathcal{E}_{2}, \mathcal{E}_{3}\right)$ to $\left(\mathcal{E}_{0}, \mathcal{E}_{2}, \mathcal{E}_{1}, \mathcal{E}_{3}\right)$. Thus, the six pairs $(U, V)=\left(1, \sigma_{i}\right)$ and $\left(e^{i \pi \sigma_{i} / 4}, e^{-i \pi \sigma_{i} / 4}\right), 1 \leqslant i \leqslant 3$, generate the tetrahedral symmetry group consisting of 4 ! maps (all permutations of the indices). In particular, four small tetrahedra $T_{\mu}$ are equivalent if we disregard unitary operations before and after the noise process.

\section{SUPPRESSION OF UNITAL NOISE IN THE CASE OF A SINGLE QUBIT}

Now we present the second main result, which is on state protection against arbitrary unital noise when $\operatorname{dim} \mathcal{H}=2$. The theorem extends the result for the depolarizing noise [17] to general unital noise.

In general, there is a trade-off between the information gained and the disturbance caused by the ex-ante control. Thus one usually expects that a protocol with weak ex-ante measurements and weak ex-post control is optimal. However, the theorem states that this is not the case. As was explained in the previous section, we may assume [30] that a unital noise channel $\mathcal{N}$ is contained in a tetrahedron $T$ with vertices at unitary operations $\mathcal{E}_{0}(1,1,1), \mathcal{E}_{1}(1,-1,-1), \mathcal{E}_{2}(-1,1,-1)$ (see Fig. 2). The channel $\mathcal{N} \in T$ is expressed in the coordinate system $\left(d^{i}\right)$ specified by (21), or equivalently by

$$
\mathcal{N}\left(\frac{1+\sum_{i} x^{i} \sigma_{i}}{2}\right)=\frac{1+\sum_{i} d^{i} x^{i} \sigma_{i}}{2} .
$$

Theorem 2. Let $\operatorname{dim} \mathcal{H}=2$ and let $\mathcal{N} \in T$. The optimal exante-ex-post control protocol $\left\{\left(\mathcal{J}_{\omega}, \mathrm{C}_{\omega}\right)\right\}_{\omega=1,2, \ldots}$ for suppression of $\mathcal{N}$ is given as follows.

(i) When $\mathcal{N} \in T_{\mu}$, the optimal protocol $\left\{\left(\mathcal{J}_{\omega}, \mathrm{C}_{\omega}\right)\right\}_{\omega=1}$ is a no measurement protocol defined by

$$
\mathcal{J}_{1}=\mathrm{id}, \quad \mathrm{C}_{1}=\mathcal{A}_{\sigma_{\mu}} .
$$

The optimal average fidelity is

$$
\bar{F}_{\mathrm{NM}}=\frac{1}{2}+\frac{\left|d^{1}\right|+\left|d^{2}\right|+\left|d^{3}\right|}{6} .
$$

(ii) When $\mathcal{N} \in O$, the discriminate and reprepare protocol $\left\{\left(\mathcal{J}_{\omega}, \mathrm{C}_{\omega}\right)\right\}_{\omega=1,2}$, defined by (8) and (9) with $d=2$, is optimal. The optimal average fidelity is $\bar{F}_{\mathrm{DR}}=2 / 3$.

The no measurement protocol above does not involve any measurement and merely cancels the reversible part of the noise. When $\mu=0$, it is nothing but the do nothing protocol and the value of the average fidelity $\bar{F}_{\mathrm{DN}}$ can be obtained by direct calculation,

$$
\begin{aligned}
\bar{F}_{\mathrm{DN}} & =\int \frac{d S}{4 \pi} \operatorname{Tr}\left[\frac{1+\sum_{i} x^{i} \sigma_{i}}{2} \frac{1+\sum_{i} d^{i} x^{i} \sigma_{i}}{2}\right] \\
& =\frac{1}{2}+\frac{\sum_{i} d^{i}}{6},
\end{aligned}
$$

where the integral is over the sphere $\|\boldsymbol{x}\|=1$ and $d S$ is the area element. The discriminate and reprepare protocol appeared in Proposition 1 and $\bar{F}_{\mathrm{DR}}=2 / 3$ follows from Eq. (10) and $\operatorname{dim} \mathcal{H}=2$. The no measurement and discriminate and reprepare protocols are considered "classical" because one either performs no quantum measurement at all or only uses the classical information extracted by the ex-ante measurement.

The difference between noise in $T_{\mu}$ and $O$ can be understood by the strength of noise. In fact, the vertices $\mathcal{E}_{\mu}$ are unitary operations, while the origin, which always outputs the completely mixed state, entirely destroys the initial state. The theorem above states that the optimal protocol depends on the strength of the noise and suddenly changes at the threshold, with no intermediate regime in which truly quantum protocols are optimal.

Lemma 1. In the two-dimensional Hilbert space $\mathcal{H}$, consider the noise $\mathcal{N}=\mathcal{E}_{0 i}, 1 \leqslant i \leqslant 3$, i.e.,

$$
\mathcal{N}(\rho)=\frac{1}{2}\left(\rho+\sigma_{i} \rho \sigma_{i}\right) .
$$

The do nothing and the discriminate and reprepare protocols are optimal ex-ante-ex-post control protocols to suppress $\mathcal{N}=$ $\mathcal{E}_{0 i}$. The optimal average fidelity is $\bar{F}=2 / 3$.

Proof. We give a proof for $i=3$; the cases $i=1,2$ are essentially the same. Let us show that the dephasing noise $\mathcal{N}=\mathcal{E}_{03}$ is a QCQ channel. Let $P_{0}$ and $P_{1}$ be the projection to the eigenspaces of $\sigma_{3}$ with eigenvalues 1 and -1 . Then, inserting $\sigma_{0}=P_{0}+P_{1}$ and $\sigma_{3}=P_{0}-P_{1}$ into $\mathcal{N}(\rho)=\frac{1}{2}\left(\sigma_{0} \rho \sigma_{0}+\sigma_{3} \rho \sigma_{3}\right)$, one can rewrite $\mathcal{N}$ in the form (7) with $\rho_{k}:=P_{k}$ and $M_{k}:=P_{k}, k=0,1$. Then, the optimality of the discriminate and reprepare protocol in suppressing $\mathcal{N}$ follows from Proposition 1. On the other hand, the value $\bar{F}=2 / 3$ can be attained also by the do nothing protocol, which can be seen by substituting $\left(d^{i}\right)=(0,0,1)$ into $\bar{F}_{\mathrm{DN}}$ in (25). Therefore the claim is true. 
We give in the Appendix an alternative proof of Lemma 1 which does not depend on Proposition 1 and is based on a direct calculation. Now, let us prove Theorem 2.

Proof of Theorem 2. (i) When $\mathcal{N} \in T_{0}$, it is trivial that the do nothing protocol $\left\{\left(\mathcal{J}_{\omega}, \mathcal{C}_{\omega}\right)\right\}_{\omega=1}=\{$ (id,id) $\}$ optimally suppresses the noise $\mathcal{E}_{0}=$ id with $\bar{F}=1$. From Lemma 1 , this protocol also suppresses optimally the noise $\varepsilon_{0 i}, 1 \leqslant i \leqslant 3$. Then, from Lemma 2 , the do nothing protocol optimally suppresses any noise $\mathcal{N}$ in the convex hull $T_{0}$ of $\mathcal{E}_{0}, \mathcal{E}_{01}, \mathcal{E}_{02}$, and $\varepsilon_{03}$. The average fidelity $\bar{F}$ is given by Eq. (25).

When $\mathcal{N} \in T_{i}$, the noise $\mathcal{A}_{\sigma_{i}} \circ \mathcal{N}$ is in $T_{0}$, as explained in the preceding section, and hence optimally suppressed by the do nothing protocol $\{(\mathrm{id}, \mathrm{id})\}$. Therefore, $\mathcal{N}$ here is optimally suppressed by $\left\{\left(\mathrm{id}, \mathcal{A}_{\sigma_{i}}\right)\right\}$ [30], which is a no measurement protocol. The average fidelity $\bar{F}$ is given by Eq. (25) with $d^{j}(j \neq i)$ replaced with $-d^{j}$.

(ii) By Lemma 1, three of the vertices $\varepsilon_{0 i}$ of the octahedron $\mathrm{O}$ are optimally suppressed by the discriminate and reprepare protocol $\left\{\left(\mathcal{J}_{\omega}, \mathcal{C}_{\omega}\right)\right\}$ defined by (8) and (9). The other vertices can be flipped to one of the former three by $\mathcal{A}_{\sigma_{i}}$, as explained in the preceding section. Thus they are optimally suppressed by the discriminate and reprepare protocol $\left\{\left(\mathcal{J}_{\omega}, \mathrm{C}_{\omega} \circ \mathcal{A}_{\sigma_{i}}\right)\right\}[30]$. Furthermore, the protocol $\left\{\left(\mathcal{J}_{\omega}, \mathrm{C}_{\omega}\right)\right\}$ gives the same average fidelity as $\left\{\left(\mathcal{J}_{\omega}, \mathcal{C}_{\omega} \circ \mathcal{A}_{\sigma_{i}}\right)\right\}$, because the average operation (5) yields $\mathcal{E}=\sum_{\omega} \mathcal{C}_{\omega} \circ \mathcal{J}_{\omega}$ for both protocols. Thus all vertices of $\mathrm{O}$ are optimally suppressed by the same discriminate and reprepare protocol $\left\{\left(\mathcal{J}_{\omega}, \mathrm{e}_{\omega}\right)\right\}$. Recalling that $O$ is the convex hull of these six vertices, one concludes, by Proposition 2, that each $\mathcal{N} \in \mathrm{O}$ is optimally suppressed by the discriminate and reprepare protocol $\left\{\left(\mathcal{J}_{\omega}, \mathrm{e}_{\omega}\right)\right\}$.

\section{CONCLUSION AND DISCUSSIONS}

We have discussed the problem of protecting a completely unknown state against given unital noise by an ex-ante and ex-post control scheme. A protocol in the scheme is described mathematically by a family of pairs, $\left\{\left(\mathcal{J}_{\omega}, \mathrm{e}_{\omega}\right)\right\}_{\omega \in \Omega}$, where $\left\{\mathcal{J}_{\omega}\right\}_{\omega \in \Omega}$ is the CP instrument with the set $\Omega$ of outcomes which describes the ex-ante measurement and the map $\mathcal{C}_{\omega}$ is the TPCP map which describes the ex-post operation when the outcome $\omega$ is obtained. To evaluate the closeness of the input and output states, we have chosen the average fidelity $\bar{F}$ between the input and output states.

We generalized the previous results in Ref. [17] in two directions. First, we proved the previous conjecture as Theorem 1, which states that the depolarizing noise in any finite-dimensional system is optimally suppressed by classical protocols. Namely, the do nothing protocol is optimal if the noise is weak and the discriminate and reprepare protocol is optimal if the noise is strong. Then, we focused on the case of a qubit system and generalized the result to the class of unital noise, which contains many types of noise including dephasing noise, bit-flip noise, and depolarizing noise. We have proved that arbitrary unital noise is optimally suppressed by the classical protocols, namely, the no measurement protocol or the discriminate and reprepare protocol depending on the strength of the noise (Theorem 2).

Our results suggest that one can perform nontrivial suppression of noise only by taking advantage of the bias of the noise. This gives a natural understanding for the previously known facts and numerical evidence that nontrivial suppression is possible against amplitude damping noise but is not possible against depolarizing noise [17,22]. For a deeper and more precise understanding of state protection in this direction, it will be necessary to examine our Theorem 2 in higher-dimensional Hilbert spaces and to investigate optimal ex-ante-ex-post control protocols against nonunital noise.

We would like to emphasize that the method based on Propositions 1 and 2 is much more general than the previous one [17], which involved a detailed estimation of a function of several variables. Proposition 1 states that QCQ noise is optimally suppressed by the discriminate and reprepare protocol. Proposition 2 points out the convexity of the noise channels that are optimally suppressed by a single protocol. The proofs of Theorems 1 and 2 were to find several noise channels (TPCP maps) which are optimally suppressed by a single protocol and to derive the optimality of the protocol in their convex hull. It was especially important to find the particular noise channel that is optimally suppressed by two or more different protocols simultaneously, such as $\varepsilon_{\mu \nu}$ in the proof of Theorem 2. In other words, it is essential to find the watersheds (critical points) in the space of noise for the determination of the basin of optimality (convex domain) of a protocol. Further applications of the method may reveal the "phase diagram" of optimality in the space of noise.

In closing, we would like to comment on the possibility of an information-theoretic approach to our problem. As stated in Proposition 1, classical protocols suffice for suppression of the QCQ noise. Intuitively, the reason is that a QCQ noise channel changes the quantum state into a classical state (probability density) once. Besides being entanglement breaking, as mentioned in Sec. IV, a QCQ channel has zero quantum capacity (see, e.g., Ref. [31]). Since quantum capacity is the highest rate at which quantum information can be transmitted coherently, a QCQ channel can be said to transmit classical information only. This fact and our result suggest that QCQ channels are particularly "noisy." Toward solving the state protection problem in general, it may be a good idea to seek for relations between the maximal average fidelity and information-theoretic quantities such as quantum capacity and entanglement measures. We hope that further investigations in this direction open up a way of treating the problem systematically.

\section{ACKNOWLEDGMENTS}

H.W. acknowledges the support from Keio University Doctorate Student Grant-in-Aid Program. T.K. acknowledges the support from MEXT-Supported Program for the Strategic Research Foundation at Private Universities "Topological Science" and from Keio University Creativity Initiative "Quantum Community."

\section{APPENDIX: AN ELEMENTARY PROOF OF LEMMA 1}

We give a proof for the case $i=3$, the dephasing noise. The cases $i=1,2$ are similar. From Eq. (6), the optimal protocol 
$\left\{\left(\mathcal{J}_{\omega}, \mathrm{C}_{\omega}\right)\right\}_{1 \leqslant \omega \leqslant d}$ is the maximizer of $\sum_{\omega} f_{\omega}$, with

$$
\begin{aligned}
f_{\omega} & :=\operatorname{Tr}_{\mathrm{HS}} \mathcal{C}_{\omega} \circ \mathcal{N} \circ \mathcal{J}_{\omega}=\operatorname{Tr}_{\mathrm{HS}} \mathcal{N} \circ \mathcal{J}_{\omega} \circ \mathcal{C}_{\omega} \\
& =\frac{1}{2} \operatorname{Tr} \mathcal{J}_{\omega} \circ \mathcal{C}_{\omega}(1)+\frac{1}{2} \operatorname{Tr} \sigma_{z} \mathcal{J}_{\omega} \circ \mathcal{C}_{\omega}\left(\sigma_{z}\right) \\
& =\frac{1}{2} \operatorname{Tr} \mathcal{J}_{\omega}^{*}(1) \mathcal{C}_{\omega}(1)+\frac{1}{2} \operatorname{Tr} \mathcal{J}_{\omega}^{*}\left(\sigma_{z}\right) \mathcal{C}_{\omega}\left(\sigma_{z}\right),
\end{aligned}
$$

where we have used (3) and $\mathcal{N}^{*}(1)=1, \mathcal{N}^{*}\left(\sigma_{z}\right)=\sigma_{z}$, $\mathcal{N}^{*}\left(\sigma_{x}\right)=\mathcal{N}^{*}\left(\sigma_{y}\right)=0$, which follow from (26).

Let us write

$$
\begin{array}{r}
\mathcal{C}_{\omega}(1)=1+\boldsymbol{\alpha}_{\omega} \cdot \boldsymbol{\sigma}, \quad \mathrm{C}_{\omega}\left(\sigma_{z}\right)=\boldsymbol{\beta}_{\omega} \cdot \boldsymbol{\sigma}, \\
\mathrm{J}_{\omega}^{*}(1)=\gamma_{\omega}+\boldsymbol{\delta}_{\omega} \cdot \boldsymbol{\sigma}, \quad \mathrm{J}_{\omega}^{*}\left(\sigma_{z}\right)=\varepsilon_{\omega}+\boldsymbol{\zeta}_{\omega} \cdot \boldsymbol{\sigma} .
\end{array}
$$

Because $v+\xi \cdot \sigma \geqslant 0$ holds if and only if $\|\xi\| \leqslant v$, the positivity of $\mathcal{C}_{\omega}$ and $\mathcal{J}_{\omega}$ implies $\left\|\boldsymbol{\alpha}_{\omega} \pm \boldsymbol{\beta}_{\omega}\right\| \leqslant 1$ and $\left\|\boldsymbol{\delta}_{\omega} \pm \boldsymbol{\zeta}_{\omega}\right\| \leqslant$ $\gamma_{\omega} \pm \varepsilon_{\omega}$, respectively (consider the images of $1 \pm \sigma_{z}$ ). One therefore has

$$
\begin{aligned}
f_{\omega} & =\gamma_{\omega}+\boldsymbol{\alpha}_{\omega} \cdot \boldsymbol{\delta}_{\omega}+\boldsymbol{\beta}_{\omega} \cdot \boldsymbol{\zeta}_{\omega} \\
& =\gamma_{\omega}+\frac{1}{2}\left[\left(\boldsymbol{\alpha}_{\omega}+\boldsymbol{\beta}_{\omega}\right) \cdot\left(\boldsymbol{\delta}_{\omega}+\boldsymbol{\zeta}_{\omega}\right)+\left(\boldsymbol{\alpha}_{\omega}-\boldsymbol{\beta}_{\omega}\right) \cdot\left(\boldsymbol{\delta}_{\omega}-\boldsymbol{\zeta}_{\omega}\right)\right] \\
& \leqslant \gamma_{\omega}+\frac{1}{2}\left(\left\|\boldsymbol{\alpha}_{\omega}+\boldsymbol{\beta}_{\omega}\right\|\left\|\boldsymbol{\delta}_{\omega}+\boldsymbol{\zeta}_{\omega}\right\|+\left\|\boldsymbol{\alpha}_{\omega}-\boldsymbol{\beta}_{\omega}\right\|\left\|\boldsymbol{\delta}_{\omega}-\boldsymbol{\zeta}_{\omega}\right\|\right) \\
& \leqslant \gamma_{\omega}+\frac{1}{2}\left[\left(\gamma_{\omega}+\varepsilon_{\omega}\right)+\left(\gamma_{\omega}-\varepsilon_{\omega}\right)\right] \\
& =2 \gamma_{\omega},
\end{aligned}
$$

where we have used the Cauchy-Schwarz inequality in the first inequality. It follows from trace preservation of $\sum_{\omega} \mathcal{J}_{\omega}$, or $\sum_{\omega} \mathcal{J}_{\omega}^{*}(1)=1$, that $\sum_{\omega} \gamma_{\omega}=1$ holds. As a result, one obtains $\sum_{\omega} f_{\omega} \leqslant 2$, which is, from Eq. (6), equivalent to

$$
\bar{F} \leqslant \frac{2}{3} \text {. }
$$

This value is attained by both the do nothing and the discriminate and reprepare protocols, which can be seen by (25) with $\left(d^{1}, d^{2}, d^{3}\right)=(1,0,0)$ and by $(10)$, respectively.
[1] P. W. Shor, Phys. Rev. A 52, R2493(R) (1995).

[2] E. Knill and R. Laflamme, Phys. Rev. A 55, 900 (1997).

[3] M. Reimpell and R. F. Werner, Phys. Rev. Lett. 94, 080501 (2005); N. Yamamoto, S. Hara, and K. Tsumura, Phys. Rev. A 71, 022322 (2005).

[4] D. A. Lidar, I. L. Chuang, and K. B. Whaley, Phys. Rev. Lett. 81, 2594 (1998)

[5] L. Viola, E. Knill, and S. Lloyd, Phys. Rev. Lett. 82, 2417 (1999).

[6] E. B. Davies and J. T. Lewis, Commun. Math. Phys. 17, 239 (1970).

[7] M. Ozawa, J. Math. Phys. 25, 79 (1984).

[8] C. W. Helstrom, Inf. Control 10, 254 (1967).

[9] I. D. Ivanovic, Phys. Lett. A 123, 257 (1987); D. Dieks, ibid. 126, 303 (1988); A. Peres, ibid. 128, 19 (1988).

[10] A. Chefles, Phys. Lett. A 239, 339 (1998).

[11] R. Kawakubo and T. Koike, J. Phys. A: Math. Theor. 49, 265201 (2016).

[12] W. K. Wooters and W. H. Zurek, Nature (London) 299, 802 (1982); D. Dieks, Phys. Lett. A 92, 271 (1982); H. P. Yuen, ibid. 113, 405 (1986).

[13] M. Ozawa, Phys. Rev. A 67, 042105 (2003).

[14] Y. Watanabe, T. Sagawa, and M. Ueda, Phys. Rev. A 84, 042121 (2011).

[15] V. Bužek and M. Hillery, Phys. Rev. A 54, 1844 (1996).

[16] R. F. Werner, Phys. Rev. A 58, 1827 (1998).
[17] H. Wakamura, R. Kawakubo, and T. Koike, Phys. Rev. A 95 022321 (2017).

[18] A. M. Brańczyk, P. E. M. F. Mendonça, A. Gilchrist, A. C. Doherty, and S. D. Bartlett, Phys. Rev. A 75, 012329 (2007).

[19] P. E. M. F. Mendonça, A. Gilchrist, and A. C. Doherty, Phys. Rev. A 78, 012319 (2008).

[20] S. Zhang, X. Zou, C. Li, C. Jin, and G. Guo, arXiv:0811.3254.

[21] A. N. Korotkov and K. Keane, Phys. Rev. A 81, 040103 (2010).

[22] C. Q. Wang, B. M. Xu, J. Zou, Z. He, Y. Yan, J. G. Li, and B. Shao, Phys. Rev. A 89, 032303 (2014).

[23] M. A. Nielsen and I. L. Chuang, Quantum Computation and Quantum Information (Cambridge University Press, Cambridge, U.K., 2000).

[24] A. S. Holevo, Russ. Math. Surv. 53, 1295 (1998).

[25] P. W. Shor, J. Math. Phys. 43, 4334 (2002).

[26] M. Horodecki, P. Shor, and M. B. Ruskai, Rev. Math. Phys. 15, 629 (2003).

[27] D. Bruß and C. Macchiavello, Phys. Lett. A 253, 249 (1999).

[28] G. Vidal and R. Tarrach, Phys. Rev. A 59, 141 (1999).

[29] C. King and M. B. Ruskai, IEEE Trans. Inf. Theory 47, 192 (2001).

[30] The optimal protocol $\left\{\left(\mathcal{J}_{\omega}^{\prime}, \mathrm{C}_{\omega}^{\prime}\right)\right\}$ for noise $\mathcal{N}^{\prime}$ of the form (19) is obtained from the optimal protocol $\left\{\left(\mathcal{J}_{\omega}, \mathcal{C}_{\omega}\right)\right\}$ for $\mathcal{N}$ by setting $\mathrm{J}_{\omega}^{\prime}:=\mathcal{A}_{U^{\dagger}} \circ \mathcal{J}_{\omega}$ and $\mathcal{C}_{\omega}^{\prime}:=\mathcal{C}_{\omega} \circ \mathcal{A}_{V^{\dagger}}$.

[31] T. S. Cubitt, M. B. Ruskai, and G. Smith, J. Math. Phys. 49, 102104 (2008). 